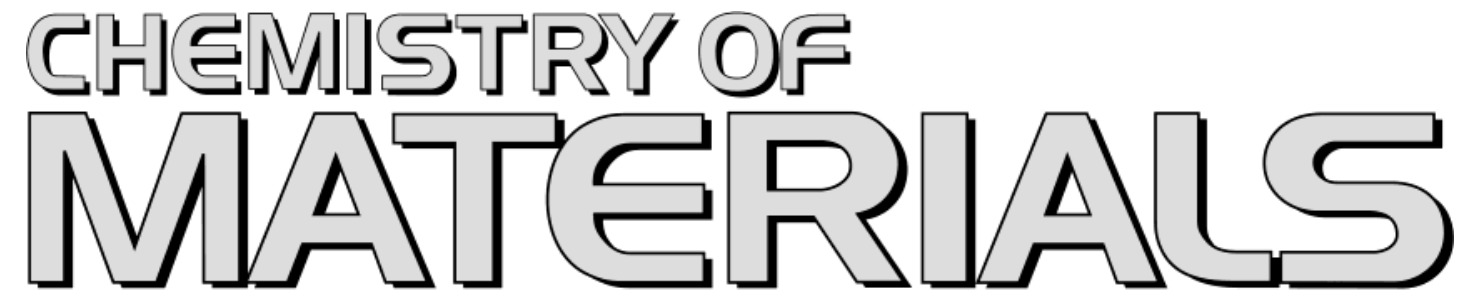

VOLUME 16, NUMBER 4

FEBRUARY 24, 2004

(C) Copyright 2004 by the American Chemical Society

\title{
Communications
}

\section{Fabrication of Tunable Superhydrophobic Surfaces by Nanosphere Lithography}

\author{
J au-Ye Shiu, ${ }^{\dagger}$ Chun-Wen Kuo, ${ }^{\dagger}$ Peilin Chen, ${ }^{*},{ }^{\dagger}$ and \\ Chung-Yuan $\mathrm{Mou}^{\ddagger}$
}

Institute of Applied Science and Engineering Research, Academia Sinica, 128, Section 2, Academia Road, Nankang, Taipei 115, Taiwan, and Department of Chemistry, National Taiwan University, Taipe, Taiwan

Received J uly 28, 2003

Revised Manuscript Received December 16, 2003

Inspired by the water-repellent behavior of the microand nanostructured plant surfaces, ${ }^{1,2}$ superhydrophobic materials, with a water contact larger than $150^{\circ}$, have received a lot of research attention recently..$^{3-17}$ It has been suggested that contamination, oxidation, and current conduction can be inhibited on such superhydrophobic surfaces, ${ }^{18}$ and the flow resistance in the microfluidic channels can also be reduced using super water-repellent materials. ${ }^{19}$ However, to fully utilize the

* Corresponding author. E-mail: peilin@gate.sinica.edu.tw. Tel: +886-2-2789-8000. Fax: +886-2-2782-6680.

† Academia Sinica.

¥ National Taiwan University.

(1) Neinhuis, C.; Barthlott, W. Ann. Bot. 1997, 79, 677.

(2) Barthlott, W.; Neinhuis, C. Planta 1997, 202, 1.

(3) Feng, L.; Li, S.; Li, Y.; Li, H.; Zhang, L.; Zhai, J .; Song, Y.; Liu, B.; J iang, L.; Zhu, D. Adv. Mater. 2002, 14, 1857.

(4) Gu, Z.-Z.; Uetsuka, H.; Takahashi, K.; Nakajima, R.; Onishi, H.; Fujishima, A.; Sato, O. Angew. Chem., Int. Ed. 2003, 42, 894.

(5) Teare, D. O. H.; Spanos, C. G.; Ridley, P.; Kinmond, E. J .; Roucoules, V.; Badyal, J. P. S. Chem. Mater. 2002, 14, 4566.

(6) Tsujii, K.; Yamamoto, T.; Onda, T.; Shibuchi, S. Angew. Chem., Int. Ed. 1997, 36, 1001.

(7) Erbil, H. Y.; Demirel, A. L.; Avci, Y.; Mert, O. Science 2003, 299, 1377.

(8) Woodward, I.; Schofield, W. C. E.; Roucoules, V.; Badyal, J . P. S. Langmuir 2003, 19, 3432.

(9) Morra, M.; Occhiello, E.; Garbassi, F. Langmuir 1989, 5, 872. water-repellent properties of the nanostructured surfaces, it is necessary to investigate the relationship between the nanostructure and the water repellent behavior on surfaces and to fabricate the nanostructured surfaces with desired surface hydrophobicity. In this letter, we describe a simple fabrication method for creating well-ordered nanostructured surfaces whose surface hydrophobicity can be modeled and tuned.

To fabricate superhydrophobic surfaces, a typical procedure is to create a rough surface covered with low surface energy molecules, such as fluoroalkylsilanes, ${ }^{3}$ or to roughen the surface of hydrophobic materials. Several superhydrophobic surfaces have been prepared by these approaches including fluoroalkylsilane-modified inverse opal surfaces, ${ }^{4}$ plasma polymerization, ${ }^{5}$ anodic oxidation of aluminum, ${ }^{6}$ gel-like roughened polypropylene, ${ }^{7}$ plasma fluorination of polybutadiene, ${ }^{8}$ oxygen plasma-treated polytetrefluoroethylene, ${ }^{9,10}$ densely packed aligned carbon nanotubes, ${ }^{11}$ al igned polyacrylonitile nanofibers, ${ }^{12}$ and solidification of alkylketene dimmer. ${ }^{13} \mathrm{~A}$ common observation in these experiments is that the water contact angle increases as the surface roughness increases. However, in other experiments ${ }^{14,15}$ it has been demonstrated that smooth well-ordered

(10) Morra, M.; Occhiello, E.; Garbassi, F. J . Colloid Inerface Sci. 1989, 132, 504

(11) Li, H.; Wang, X.; Song, Y.; Liu, Y.; Li, Q.; J iang, L.; Zhu, D. Angew. Chem., Int. Ed. 2002, 40, 1743.

(12) Feng, L.; Li, S.; Li, H.; Zhai, J .; Song, Y.; J iang, L.; Zhu, D. Angew. Chem., Int. Ed. 2002, 41, 1221.

(13) Shibuichi, S.; Onda, T.; Satoh, N.; Tsujii, K. J . Phys. Chem. 1996, 100, 19512.

(14) Bico, J.; Marzolin, C.; Quere, D. Europhys. Lett. 1999, 47, 220.

(15) Yoshimitsu, Z.; Nanajima, A.; Watanabe, T.; Hashimoto, K. Langmuir 2002, 18, 5818.

(16) Chen, W.; Fadeev, A. Y.; Hsieh, M. C.; Oner, D.; Youngblood, J .; McCarthy, T. J . Langmuir 1999, 15, 3395.

(17) Oner, D.; McCarthy, T. J . Langmuir 2000, 16, 7777.

(18) Nakajima, A.; Fujishima, A.; Hashimoto, K.; Watanabe, T. Adv. Mater. 1999, 11, 1365.

(19) Patankar, N. A. Langmuir 2003, 19, 1249. 
a)

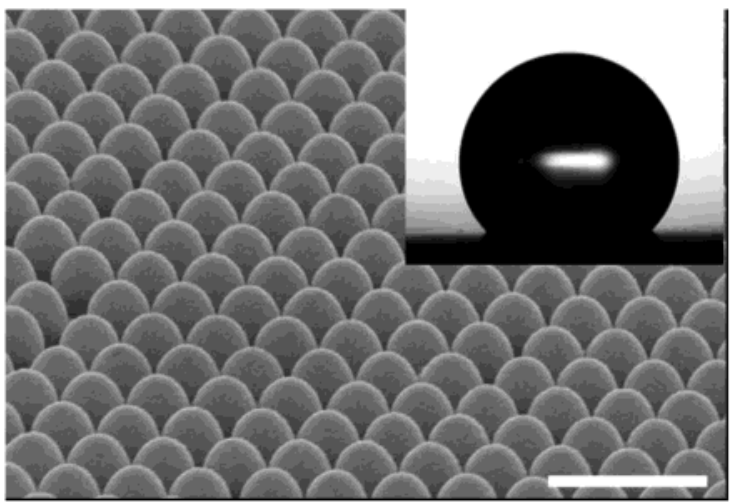

c)

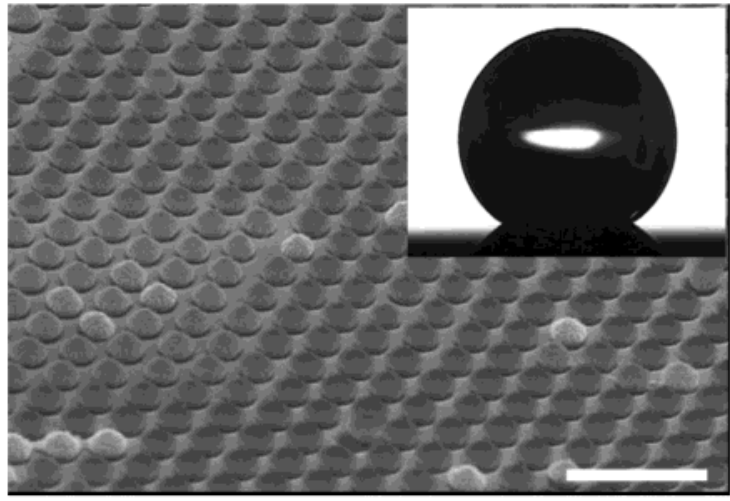

b)

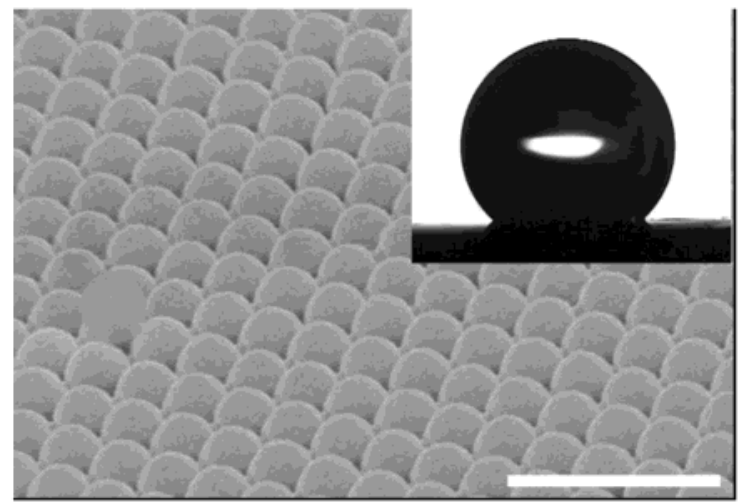

d)

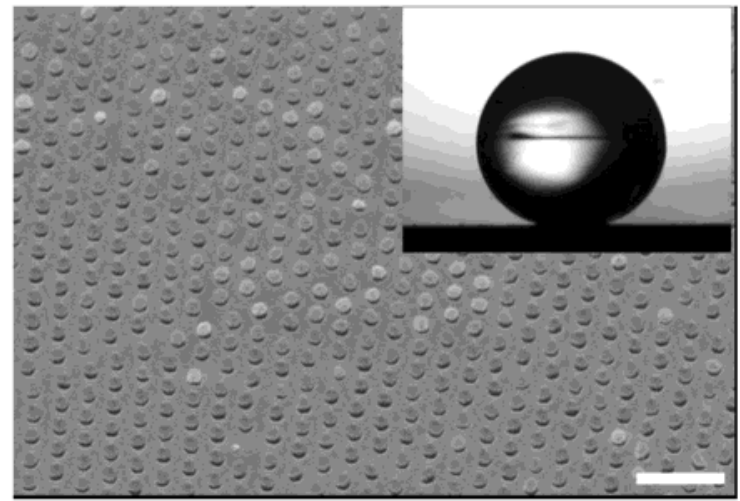

Figure 1. SEM images $\left(60^{\circ}\right)$ of the size-reduced polystyrene beads and the water contact angle measurement on the corresponding modified surfaces (insets). The diameters of polystyrene beads and water contact angles on these surfaces were measured to be (a) $400 \mathrm{~nm}, 135^{\circ}$, (b) $360 \mathrm{~nm}, 144^{\circ}$, (c) $330 \mathrm{~nm}, 152^{\circ}$, and (d) $190 \mathrm{~nm}, 168^{\circ}$. Bar: $1 \mu \mathrm{m}$.

microstructured surfaces could also produce superhydrophobic surfaces as long as the ratio of the liquidsolid contact area to the overall projected area remains small. It has also been pointed out ${ }^{16,17}$ that the threephase contact line plays a very important role in the contact angle hysteresis, which determines the sliding behavior of water droplets on surfaces. To clarify what governs the superhydrophobic behavior, we have utilized nanosphere lithography and oxygen plasma to create well-ordered nanostructured surfaces with tunable liquid-solid contact area fractions.

Nanosphere lithography 20-22 is a well-established technique for patterning large-area periodic nanosphere arrays. By spin-coating the monodisperse polystyrene beads solution on substrate surfaces, self-organized close-packed nanostructures can be easily achieved. In previous experiments, ${ }^{21-25}$ it has been shown that both single and double-layer close-packed polystyrene arrays over a few square centimeter area can be obtained by adjusting the speed of the spin-coater and the concentration of the surfactants in the polystyrene solution. After close-packed nanostructures are formed, the liquidsol id fraction of these nanostructured surfaces has been varied by oxygen plasma etching (Oxford Plasmalab 80

(20) Fischer, U. C.; Zingsheim, H. P. J . Vac. Sci. Technol. 1981, $19,881$.

(21) Hulteen, J . C.; Van Duyne, R. P. J . Vac. Sci. Technol. A 1995, 13, 1553.

(22) Haynes, C. L.; Van Duyne, R. P. J . Phys. Chem. B 2001, 105, 5599.

(23) Kuo, C.-W.; Shiu, J.-Y.; Cho, Y.-H.; Chen, P. Adv. Mater. 2003 $15,1065$.

(24) Kuo, C.-W.; Shiu, J.-Y.; Chen, P. Chem. Mater. 2003, 15, 2917.

(25) Kuo, C.-W.; Shiu, J.-Y.; Chen, P.; Somorjai, G. A. J . Phys. Chem. B 2003, 107, 9950
Plus, $130 \mathrm{~W}, 20 \mathrm{sccm} \mathrm{O}$ ), which reduced the diameter of the polystyrene beads while keeping their separation distance constant. To create water-repellent surfaces, the size-reduced polystyrene arrays were then coated with a 20-nm-thick gold film and modified with octadecanethiol (ODT, Aldrich). Figure 1 shows the scanning electron mi croscope (SE M) images of these size-reduced polystyrene arrays. In this experiment, 440-nm-diameter polystyrene beads (Bangs Laboratories) were used to form single-layer arrays. As seen from Figure 1, the diameter of polystyrene has been systematically reduced to $190 \mathrm{~nm}$ by oxygen plasma treatment. As the result of reducing the solid-liquid contact area fraction, the apparent water contact angle of the modified polystyrene surfaces changed from $132^{\circ}$ of 440 -nm-diameter polystyrene arrays (not shown in figure) to $168^{\circ}$ of $190-$ nm-diameter size-reduced polystyrene arrays (shown in Figure 1d). The water contact angles measured on the nanostructured surfaces were much larger than that of an ODT-modified gold surface on a flat substrate $\left(114^{\circ}\right)$. These results clearly indicate that it is the surface nanostructure not the low surface energy molecules that governs the superhydrophobic behavior of surfaces.

Tolearn more about superhydrophobic behavior of the nanostructured surfaces prepared by nanosphere lithography, it is necessary to model the apparent contact angle as a function of the diameter of the sizereduced polystyrene arrays. In general, there are two wellestablished models to describe the water dewetting behavior on a rough surface, which can be attributed to Wenzel ${ }^{26}$ and Cassie and Baxter. ${ }^{27}$ In Wenzel's

(26) Wenzel, R. N. Ind. Eng. Chem. 1936, 28, 988.

(27) Cassie, A. B. D.; Baxter, S. Trans. Faraday. Soc. 1944, 40, 546. 


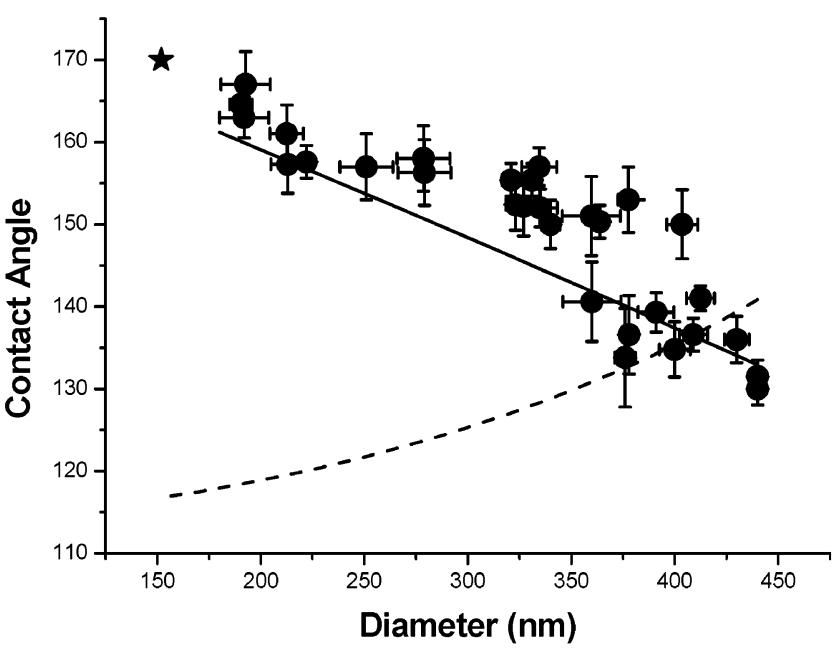

Figure 2. Measurement of the apparent water contact angle on various size-reduced polystyrene surfaces. The solid line is calculated using the modified Cassie's formulation. The dashed line is calculated by Wenzel's model. The star is the water contact angle of double-layer polystyrene arrays that underwent $120 \mathrm{~s}$ of oxygen plasma treatment.

formulation, it is assumed that the liquid fills up the rough surface, therefore forming a wetted contact, and the apparent water contact angle $\left(\theta^{*}\right)$ can be written as $\cos \theta^{*}=\gamma \cos \theta$, where $\gamma$ is the roughness factor which is the ratio of total surface area to the projected area on the horizontal plane and $\theta$ is the intrinsic contact angle measured on the flat surface. In Cassie's approach, it is assumed that the liquid forms a line of contact on the rough surface with air trapped bel ow the contact line and the apparent contact angle can be formulated as $\cos \theta^{*}=\phi_{\mathrm{s}}(\cos \theta+1)-1$, where $\phi_{\mathrm{s}}$ is the area fraction of the liquid-solid contact to the projected surface area. For our system, Wenzel's formulation predicts that the apparent water contact angle on hydrophobic surfaces will decrease as the size of polystyrene beads reduces due to the reduction of the total surface area (dashed line in Figure 2). This is in contradictory to our measurement, which shows an opposite trend (solid circles). To model our system with Cassie's formulation, it is necessary to know the area fraction above the liquid-solid contact line. Because we al ways have a hydrophobic surface, we assume that the water contact line lies on the upper part of the nanosphere. Therefore, we adopted the modified Cassie's formula for the hemispherical case in reference 14 where the apparent water contact can be written as cos $\theta^{*}=\phi_{\mathrm{B}}(\cos \theta+1)^{2}-1 ; \phi_{\mathrm{B}}$ is ratio of the cross-section area of polystyrene beads to the projected solid surface. The solid line in Figure 2 shows the calculated contact angles using the modified Cassie's formula (solid line). It is very fascinating to see that such simple assumption predicts pretty well the water dewetting behavior on the nanostructured surfaces without any adjustable parameter.

To further explore the relationship between the size of nanostructures and the superhydrophobic behavior, we have measured the apparent water contact angle of several close-packed polystyrene arrays formed by nine different sizes of monodisperse polystyrene beads with diameter ranging from 270 to $690 \mathrm{~nm}$. Within our experimental error, all the measured apparent water contact angles showed the same value $\left(131^{\circ} \pm 2^{\circ}\right)$. F rom the Wenzel's formulation, the water contact angle on these surfaces should be around $141^{\circ}$, assuming that they all have the same roughness factor $(\gamma)$, which equals 1.9 based on the close-packed sphere geometry. ${ }^{28}$ However, the apparent water contact angle calculated from the Cassie's model is around $133^{\circ}$, which agrees with our observation. This result again confirms that the Cassie's formulation provides a better description for the water dewetting behavior on such nanostructured surfaces.

Despite the successfulness of the Cassie's model in describing the superhydrophobic behavior of the nanostructured surfaces, there are still some discrepancies between the experimental observation and the Cassie's model. First, it is assumed that the liquid-solid contact linelies on the topmost layer of surface nanostructures. Therefore, the water contact angle should not be affected by the number of layers of polystyrene beads underneath the surface layer. However, the water contact angle measurement on the gold/ODT-modified doublelayer polystyrene arrays al ways possesses a larger value than those measured on the single-layer polystyrene arrays under the same oxygen etching condition. Indeed, the largest water contact angle measured in our experiment was a surface coated with double-layer polystyrene arrays prepared by $120 \mathrm{~s}$ of oxygen plasma treatment (shown in Figure 3). The difference in water contact angle between single- and doublelayer arrays may be explained by the defects formed during the nanosphere lithography process. Even though the whole substrate surfaces were covered with the close-packed polystyrene beads, there were defects between domains of wellordered polystyrene arrays, which were estimated to be less than $10 \%$ of the overall surface area. Since the water contact angle is a macroscopic property, we measured the averaged behavior of the nanostructured surfaces and the defects (which are mainly consisted of flat area) for single-layer polystyrene arrays. However, for double-layer polystyrene arrays, the surface was al ways covered with nanostructures even at defects (as judged by the SEM images). Therefore, higher contact angles were observed on doublelayer polystyrene arrays. Other important features that are not included in the modified Cassie's model are the surface fine structures caused by inhomogeneous oxygen etching and the imperfect spherical shape of the etched polystyrene beads. However, the agreement between experimental values and the modified Cassie's model seems to suggest these features are not so important in the dewetting behavior of surfaces.

It has been suggested ${ }^{15-17}$ that the water contact angle hysteresis plays an important role for the sliding behavior of water droplets. To measure the dynamic contact angle, the sessile drop technique was utilized in an experimental setup similar to a previous measure ment. ${ }^{28}$ The advancing and receding angles were obtained by increasing or decreasing the drop volume until the three-phase boundary moved over the surfaces. Both advancing and receding water contact angles are listed in Table 1 as a function of oxygen treatment time and diameter of the size-reduced polystyrene beads. The measured water contact angle hysteresis is relatively

(28) Nakae, H.; Inui, R.; Hirata, Y.; Saito, H. Acta Mater. 1998 $46,2313$. 


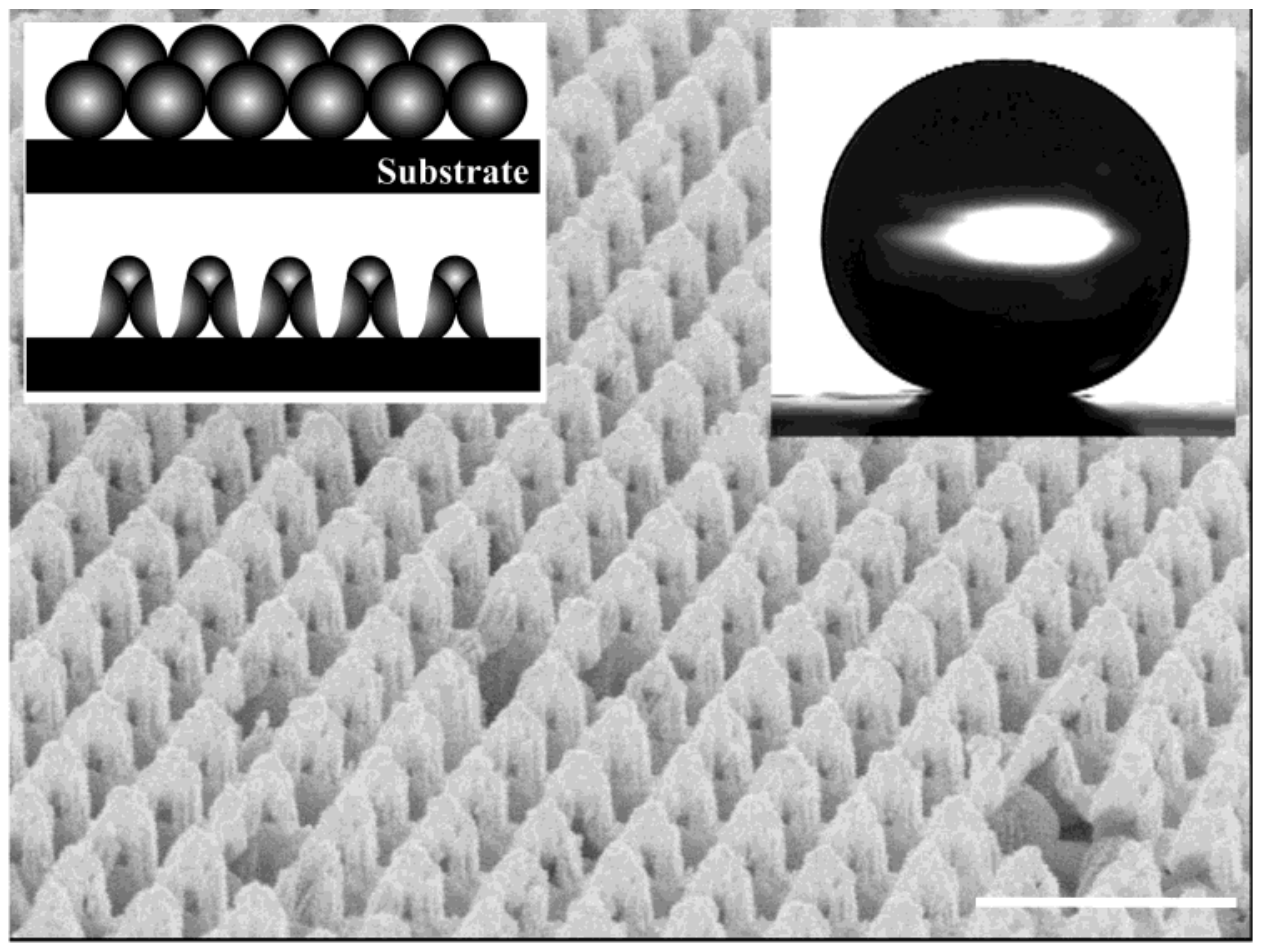

Figure 3. SEM image of a 440-nm-diameter doublelayer polystyrene surface after $120 \mathrm{~s}$ of oxygen plasma treatment. Bar: 1 $\mu \mathrm{m}$. (Left inset) The side-view illustration of the shape modification for double-layer arrays before and after the oxygen plasma treatment. (Right inset) The water contact angle on the corresponding modified surface was measured to be $170^{\circ}$.

Table 1. Dynamic Water Contact Angle Data for Oxygen Plasma-Treated Polystyrene Nanospheres

\begin{tabular}{cccc}
\hline $\begin{array}{c}\text { oxygen treatment } \\
\text { time }(\mathrm{s})\end{array}$ & $\begin{array}{c}\text { measured } \\
\text { diameter }(\mathrm{nm})\end{array}$ & $\theta_{\mathrm{A}}$ (deg) & $\theta_{\mathrm{R}}(\mathrm{deg})$ \\
\hline 0 & 440 & 130 & 110 \\
15 & 412 & 141 & 126 \\
40 & 378 & 153 & 128 \\
80 & 279 & 158 & 134 \\
100 & 213 & 161 & 130 \\
120 & 193 & 167 & 137
\end{tabular}

large. A similar hysteresis was observed by another group ${ }^{17}$ using micrometer-size square post array. The reason for such large hysteresis can be attributed to the well-ordered nature of our system. It has been suggested the three-phase contact line for such a well-ordered twodimensional system is more stable than the randomly rough surfaces, which are tortuous in three-dimension. Indeed, we observed that the water droplets remained pinned but rotated continuously on these surfaces when the surfaces were slightly tilted.

In summary, we have fabricated well-ordered, tunable superhydrophobic surface whose water contact angle can be tuned from $132^{\circ}$ to $170^{\circ}$ (on a double-layer sample) using a combination of nanosphere lithography and oxygen plasma treatment. The water contact angle on these surfaces can be modeled by the modified Cassie's formulation without any adjustable parameter. The dynamic water contact angle measurement indicates that well-ordered two-dimensional nanostructured systems have relatively large water contact angle hysteresis.

Acknowledgment. This research was supported in part by National Science Council, Taiwan, under Contract 92-2113-M-001-036.

\section{$\mathrm{CM} 034696 \mathrm{H}$}

